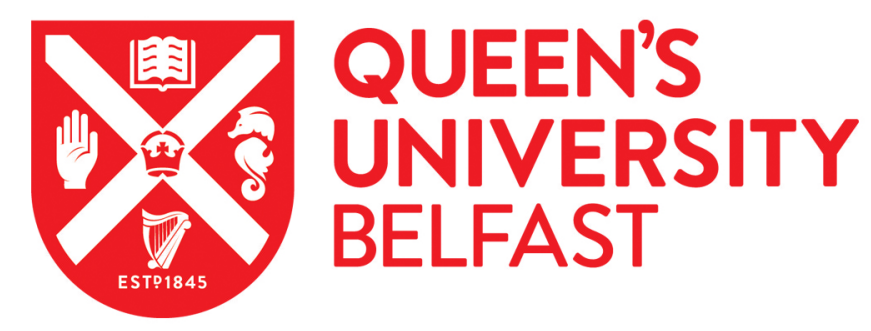

\title{
Molecular dynamics simulation investigation on the plastic flow behaviour of silicon during nanometric cutting
}

Chavoshi, S. Z., Goel, S., \& Luo, X. (2016). Molecular dynamics simulation investigation on the plastic flow behaviour of silicon during nanometric cutting. Modelling and Simulation in Materials Science and Engineering, 24(1). https://doi.org/10.1088/0965-0393/24/1/015002

Published in:

Modelling and Simulation in Materials Science and Engineering

Document Version:

Peer reviewed version

Queen's University Belfast - Research Portal:

Link to publication record in Queen's University Belfast Research Portal

Publisher rights

(c) 2016 IOP Publishing Ltd

This paper was originally published in Modelling and Simulation in Materials Science and Engineering.

\section{General rights}

Copyright for the publications made accessible via the Queen's University Belfast Research Portal is retained by the author(s) and / or other copyright owners and it is a condition of accessing these publications that users recognise and abide by the legal requirements associated with these rights.

Take down policy

The Research Portal is Queen's institutional repository that provides access to Queen's research output. Every effort has been made to ensure that content in the Research Portal does not infringe any person's rights, or applicable UK laws. If you discover content in the Research Portal that you believe breaches copyright or violates any law, please contact openaccess@qub.ac.uk. 


\title{
Molecular dynamics simulation investigation on plastic flow behaviour of silicon during nanometric cutting
}

\author{
Saeed Zare Chavoshi ${ }^{1}$, Saurav Goel $^{2}$, Xichun Luo ${ }^{1 *}$ \\ ${ }^{1}$ Centre for Precision Manufacturing, Department of Design, Manufacture and Engineering \\ Management, University of Strathclyde, Glasgow, G1 1XJ, UK \\ ${ }^{2}$ School of Mechanical and Aerospace Engineering, Queen’s University, Belfast, BT9 5AH, \\ UK \\ *Corresponding author: xichun.luo@strath.ac.uk
}

\begin{abstract}
Molecular dynamics simulation (MDS) was carried out using two types of interatomic potential function (a modified version of the Tersoff potential and an analytical bond order potential $(\mathrm{ABOP})$ ) to acquire an in-depth understanding of the material flow behaviour of single crystal silicon during nanometric cutting on three principal crystallographic planes and at different cutting temperatures. The key findings were that (i) the substrate material underneath the cutting tool was observed for the first time to experience a rotational flow akin to fluids at all the tested temperatures up to $1200 \mathrm{~K}$. The degree of flow in terms of vorticity was found higher in the (111) crystal plane signifying better machinability on this orientation in accord with the current pool of knowledge (ii) an increase in the machining temperature reduces the springback effect and thereby the elastic recovery and (iii) the cutting orientation and the cutting temperature showed significant dependence on the location and position of the stagnation region in the cutting zone of the substrate. However, a major anecdote of the study was that
\end{abstract}


irrespective of the cutting plane or the cutting temperature, the state of the cutting edge of the diamond tool did not showed symptoms of any direct diamond to graphitic phase transformation.

Keywords: Molecular dynamics; Material flow; Stagnation region; Nanometric cutting; Single crystal silicon

\section{Introduction}

An in-depth understanding of the plastic deformation mediated flow behaviour of single crystal silicon during its nanometric cutting is of important relevance to adjudge surface quality of the machined substrate. Single crystal silicon is a brittle material at ambient temperature due to the $s p^{3}$ bonding, relatively short bonding length and closely packed diamond cubic lattice structure, which makes it a difficult-to-machine material.

Despite an aggressive interest in studying nanometric cutting of silicon, there has been no theory till date suggesting material flow in different regions of the silicon substrate during its ductile-regime machining [1]. In this paper, an attempt was made to study the plastic deformation mediated flow behaviour of single crystal silicon during nanometric cutting processes. The research of this sort will help address an important research question which is at the pinnacle of nanotechnology i.e. what is the fate of brittle materials when acted upon by high deviatoric stresses and to what an extent this is sensitive to microstructure of the crystal and temperature of the substrate? In order to understand these, this study adopts molecular dynamics simulation (MDS) so as to shed some light on the material flow behaviour and flow stagnation phenomenon during nanometric cutting which hasn't received due attention in the previously made research efforts in this direction. 


\section{MD simulation details}

The MD simulations in this work were performed by using a public-domain computer code, "large-scale atomic/molecular massively parallel simulator” (LAMMPS) [2]. A schematic representation of the MD model has been shown in Fig. 1. The cutting was performed on the (010), (110) and (111) crystallographic planes and [100], [001] , [110] directions of silicon, respectively, while cubic orientation was used for the diamond cutting tool. The machining temperatures used in this investigation were $300 \mathrm{~K}, 500 \mathrm{~K}, 750 \mathrm{~K}, 850 \mathrm{~K}, 1173 \mathrm{~K}$ and $1500 \mathrm{~K}$ which was scaled using the NVE ensemble. To achieve precise results, equilibrium lattice constants were used to perform the simulations [3-4]. A modified variant of the Tersoff interatomic potential function refined by Agrawal et al. [5], which is robust in predicting the melting point of silicon and an analytical bond order potential (ABOP) proposed by Erhart and Albe [6], which is robust in accurately describing the mechanical properties of silicon, were adopted in this study. In order to determine the atomic flow field in different regions of the substrate, the atomic displacement of each atom from its initial configuration was calculated and accordingly the displacement vectors were plotted.

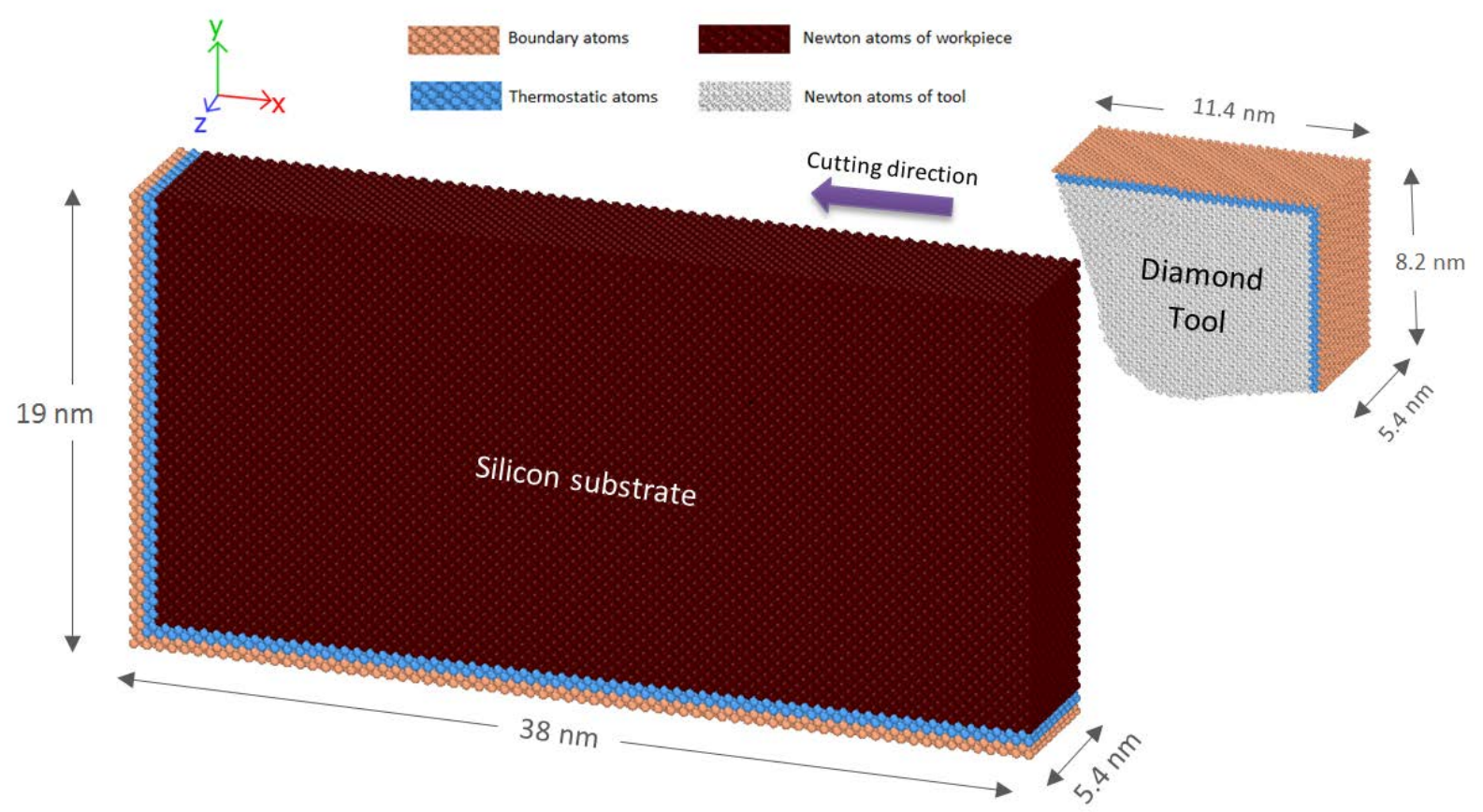


Fig. 1. Schematic representation of the MD simulation model for nanometric cutting

\section{Results and discussion}

In order to explore the atomic flow field within the substrate, the substrate was divided into five distinct regions, as shown in Fig. 2, and these were cut region, region underneath the flank face, region between the flank face and the tool tip, region ahead and beneath the tool tip and uncut region. This division was adopted for the ease of understanding as has been demonstrated later. In the stagnation region, the shearing action (leading to chip formation) separates from the compressive action underneath the cutting edge of the tool (eventually leading to springback effect) resulting in the appearance of two stagnation angles $\left(\theta_{s}\right)$ shown in Fig. 2, and this can be calculated from:

$$
\theta_{s 1,2}=\cos ^{-1}\left(1-\frac{h_{1,2}}{r}\right)
$$

where $h_{1,2}$ stands respectively for the upper and lower bounds of stagnation region, and $r$ for the tool tip radius.

A snapshot from the MD simulation obtained by modified Tersoff potential energy function after $20 \mathrm{~nm}$ of steady state cutting has been presented in Fig. 3 depicting the displacement vector of silicon atoms in the $X Y$ plane while machining at $300 \mathrm{~K}$ on the (010), (110) and the (111) crystal plane. Following conventional crystallographic convention, this paper uses ( ) and [ ] notations to represent crystallographic plane orientations (direction of plane normal) and crystallographic directions, such as cutting and slip, respectively. From Fig. 3, one can observe a rotational flow of atoms underneath the cutting tool akin to fluid flow and a distinct distribution of atomic flow field in different regions of the substrate. A same sort of atomic flow field was observed for the ABOP potential function and hence not repeated here. 


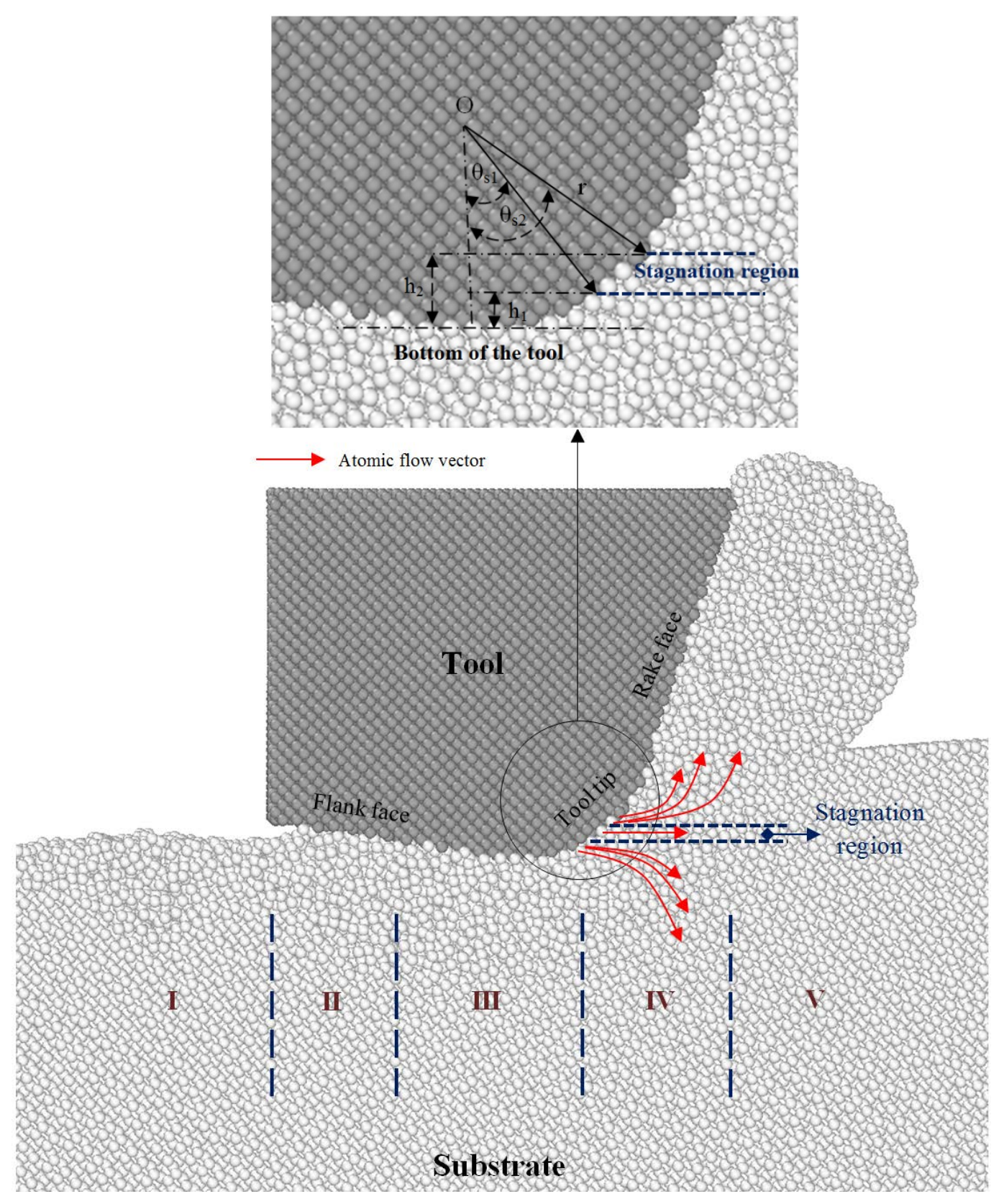

Fig. 2. Five regions in the substrate: I- Cut region, II- Underneath the flank face, III- Between the flank face and tool tip IV-Ahead and beneath the tool tip, V- Uncut region. Red arrows are flow vectors around the stagnation region. In the detailed snapshot, $\theta_{s 1,2}$ and $h_{1,2}$ represent the limiting bounds of the stagnation angles respectively in the stagnation region.

In a nanometric cutting process, there exists a so called stagnation/neutral region around the tool tip (as shown in Fig. 2) whereby the atomic flow separates into two opposite directions. The atoms above the stagnation region are separated from the substrate, and flow upward on the rake face of the cutting tool forming the cutting chips whereas atoms below the stagnation region are compressed downward by the tool tip, eventually resulting in the frequently 
observed spring back effect.
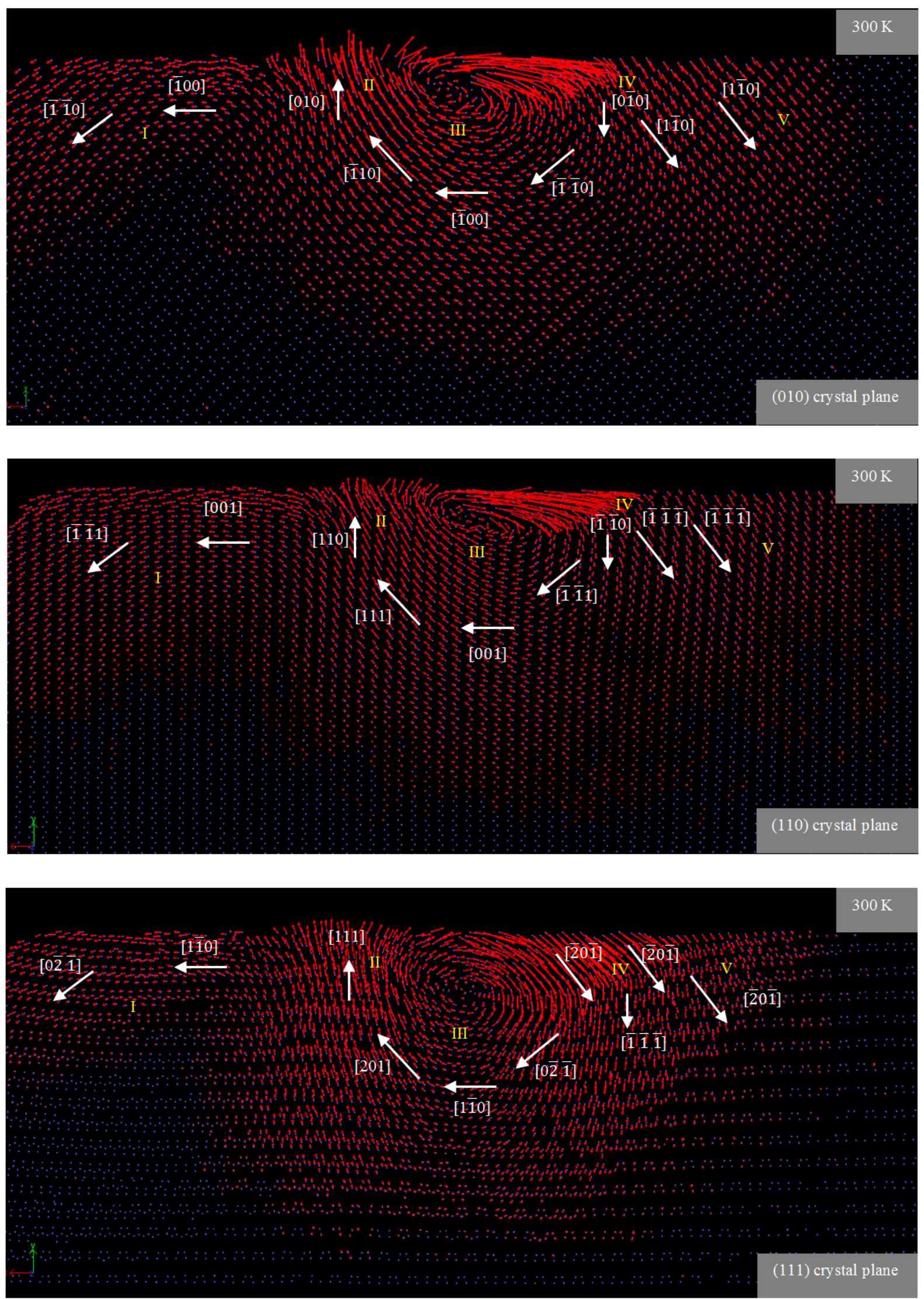
Fig. 3. Snapshots of atomic flow field in the $X Y$ plane while cutting silicon on the different crystal orientations at $300 \mathrm{~K}$. The red arrows indicate displacement vectors of atoms and their lengths demonstrate the relative magnitude of displacement. The atoms of the cutting tool are deliberately kept hidden so the position of the cutting tool with regard to the five regions in the substrate can be traced from Fig. 2.

Although shown explicitly in Fig.3, by and large, the five manifold distribution of the atomic flow field for the three crystal planes was found identical and hence only the (110) orientation has been used here for illustration purposes. The chip formation process during nanometric cutting of single crystal silicon involves extrusion of the material [7] and in this study it was

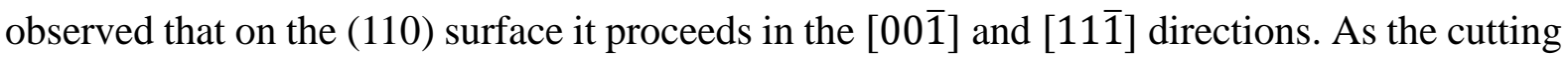
tool goes past region $I$, the highly compressed atoms in this region tend to restore their equilibrium positions in order to relieve the residual stresses. At macroscopic scale, this is also referred as material recovery or spring back effect. Hence, few distinct backward and transverse movements along the [001] and [ $\overline{1} \overline{1} 1]$ directions were observed in region $I$ during the material recovery process. As the substrate temperature increases, the magnitude of atomic displacement decreases in both the aforementioned directions, as shown in Fig. 4. In region II (underneath the flank face), the upward motion of the atoms along the [110] direction results in the elastic recovery on the flank face. As the substrate temperature increases, the movement

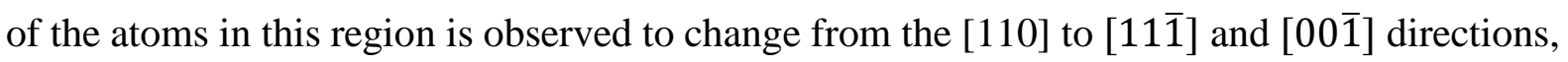
indicating reduced spring back underneath the flank face. One plausible reason for this phenomenon could be the fact that an increase of the substrate temperature results in an increase in the amplitude of atomic vibration signifying increased number of phonons which in turn contributes to additional atomic displacements. The atomic displacements within the substrate result in an increase in the interatomic distances and a decrease in the restoring forces due to thermal expansion, leading to the generation of elastic strain in the cohesive bond and corresponding lowering the energy required to break the atomic bonds. Consequently, thermal softening enhances the plasticity of the silicon substrate. 
The highly strained atoms in region $I I I$ (between the tool flank face and tool tip) under the wake of the cutting tool experience flow (site of vorticity), which comprises movement of the atoms

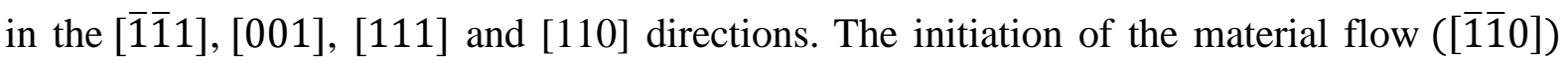
occurs underneath the lowest part of the tool tip. The centre of the flow region can be perceived as a stagnant point of the atomic flow where the theoretical displacement of the material approaches zero. Interestingly, except for $1500 \mathrm{~K}$, the vortex flow of the atoms in region III was a consistent observation at all the simulated temperatures. From a visual perspective, the flow was seemingly a laminar flow whereas at higher temperatures, such as at $1173 \mathrm{~K}$ and 1500 $\mathrm{K}$, the flow of the material was seemingly turbulent. The flow characterisation of plastically deformed material is a newly identified area of research from this paper and we shall expand on this later.

In region $I V$, the tool tip pushes the atoms downward and along the [ $\overline{1} \overline{1} \overline{1}]$ direction. It is well acknowledged that slip in a diamond cubic lattice structures occurs preferentially in the $<110>$ family of directions on the $\{111\}$ family of planes. Atoms in region $V$ move downward along the $[\overline{1} \overline{1} \overline{1}]$ direction, which is consistent with the slip system of silicon on the (111) plane. Slip in single crystal silicon is normally expected to take place on more broader spaced shuffle planes, yet micro-compression experiments at high temperatures showed that slip in silicon occurs by the movement of dislocations on the more closely spaced glide planes [8]. Fig. 5 demonstrate that when nanometric cutting was simulated using the modified Tersoff potential at $1500 \mathrm{~K}$, the substrate atoms moved along the $[00 \overline{1}]$ and [ $[\overline{1} \overline{1} \overline{1}]$ directions plausibly due to the weaker van der Waals interactions between atoms. Additionally it can also be seen that the turbulence in the substrate atoms scales linearly with the increase in the machining temperature. It was noticed that such a peculiar information about the flow at $1500 \mathrm{~K}$ was only evident from the modified Tersoff potential function and not from the ABOP function. This is on account of the fact that the modified Tersoff potential was parameterized to reproduce the melting point 
of silicon precisely, so the atomic flow behaviour at high temperatures from this potential is more reliable. Thus, it may be inferred that an interatomic potential function plays a key role in studying mechanical behaviour of materials at high temperatures.

Another key finding from Fig. 3 is that when nanometric cutting was performed on the (111) orientation, the position of the centre of rotational flow in region III was observed to be located lower than those on the other crystal planes, and thereby contributing to a more influential flow of the atoms in the substrate that facilitated the material removal process during nanometric cutting. The observed phenomenon can be considered as a confirmation for the fact that the [110] is the easy cutting direction on the (111) plane of silicon [3, 9]. In addition, by comparing the distorted atoms highlighted by grey circles in the Fig. 4, one can deduce that when hot nanometric cutting is performed on the (110) crystal plane, the number of displaced atoms will be higher than those on other crystal planes.

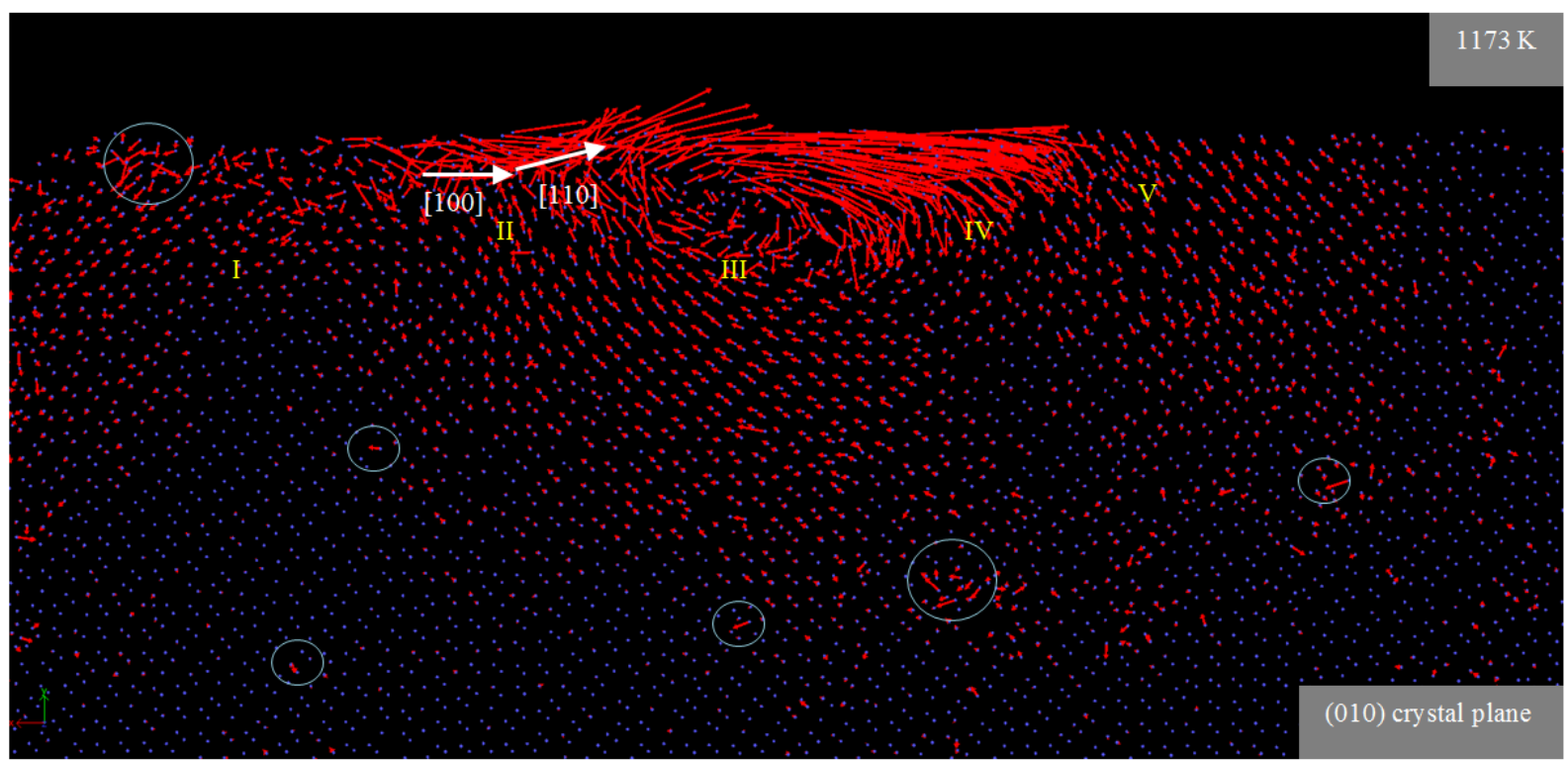



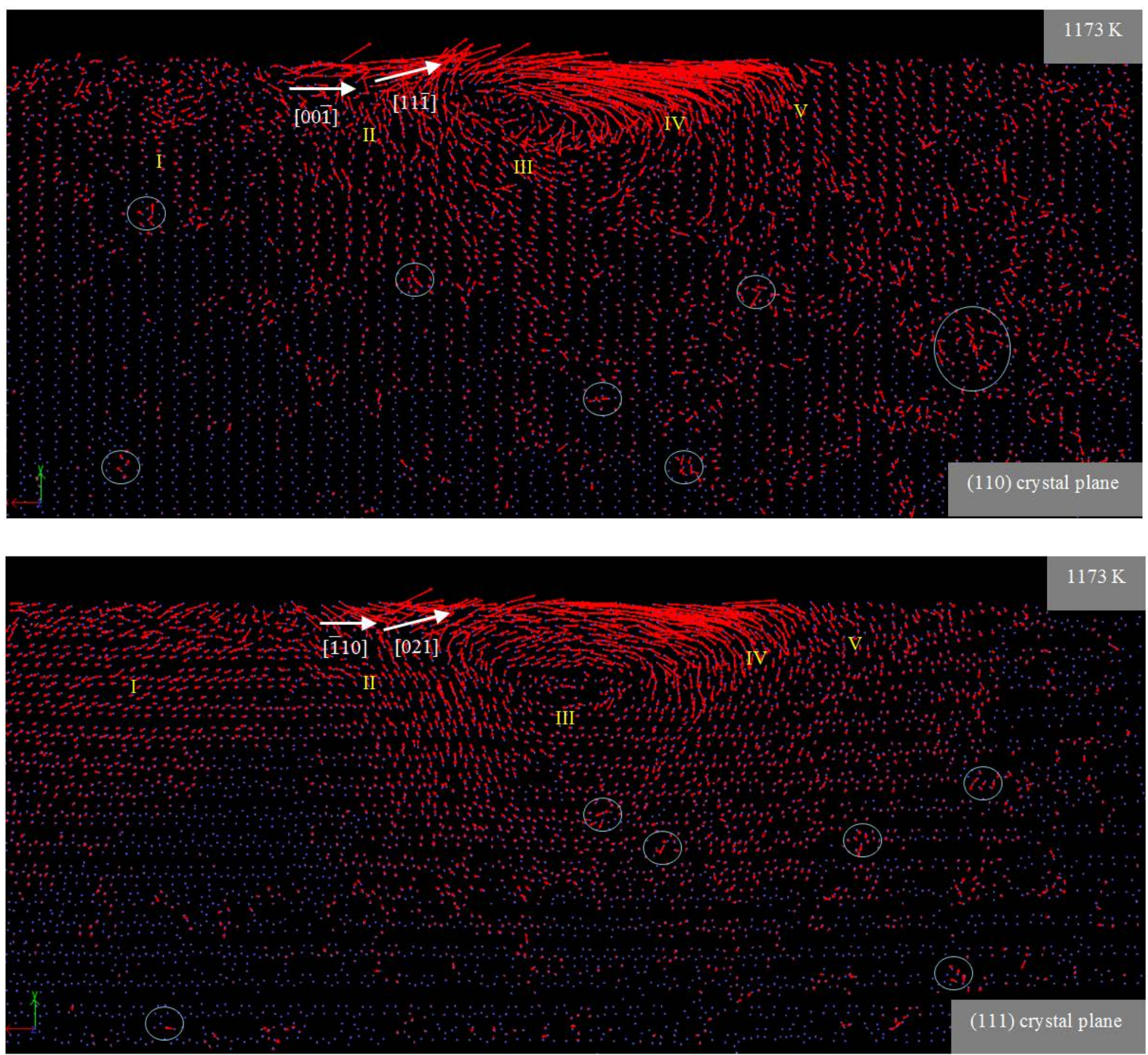

Fig. 4. Snapshots of atomic flow field while cutting silicon on the different crystal planes at $1173 \mathrm{~K}$. At high temperatures, the upward movement of atoms is eliminated, leading to decrease of the spring back on the flank face. More distorted atoms are highlighted by grey circles. 


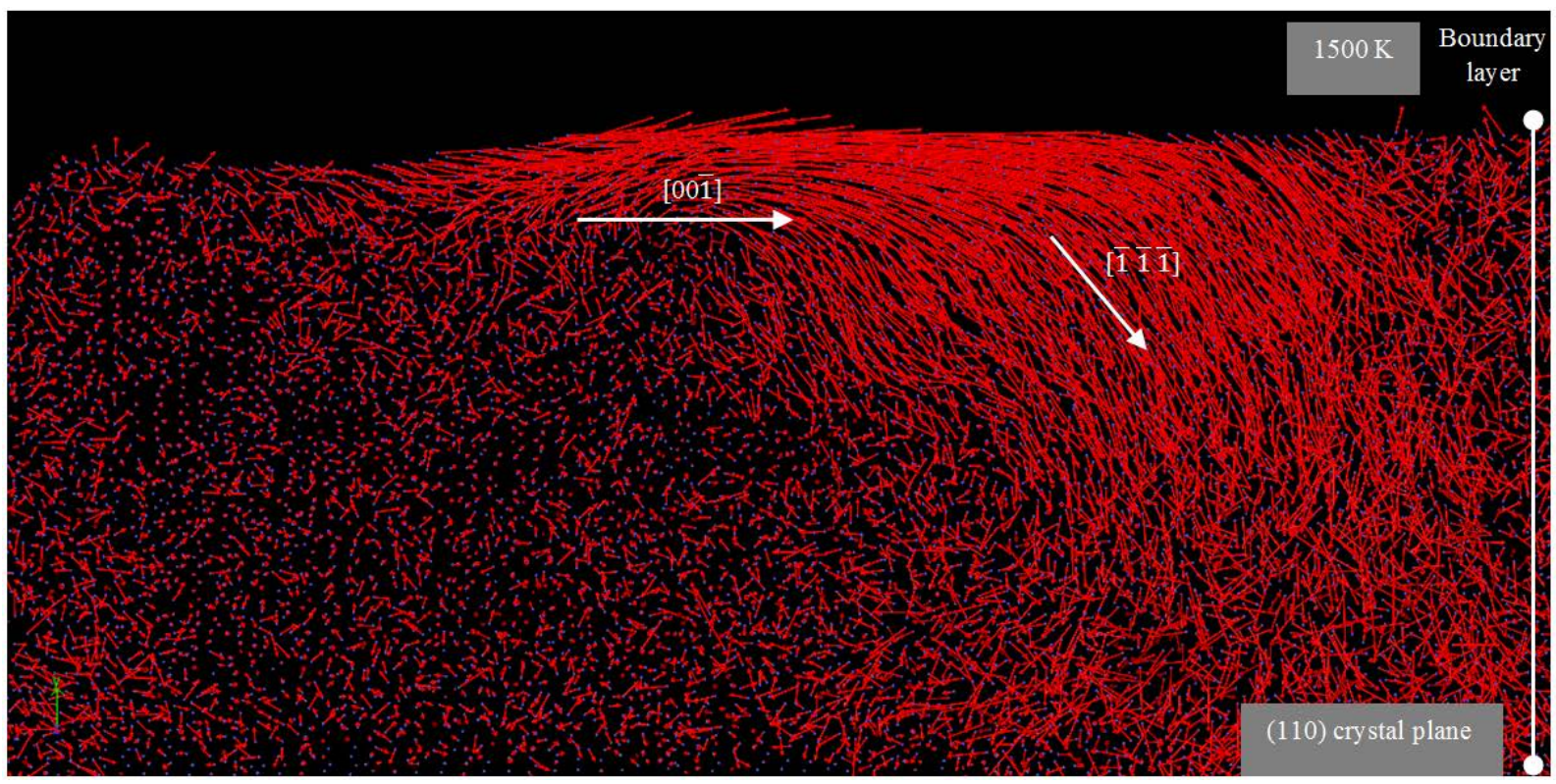

Fig. 5. Snapshot of atomic flow field while cutting silicon on the (110) crystal plane at $1500 \mathrm{~K}$ using modified Tersoff potential function. Substrate atoms move along the cutting and slip system directions owing to the very weak interaction between atoms and viscous flow of the material. Chaotic behaviour of the atoms can be observed as well.

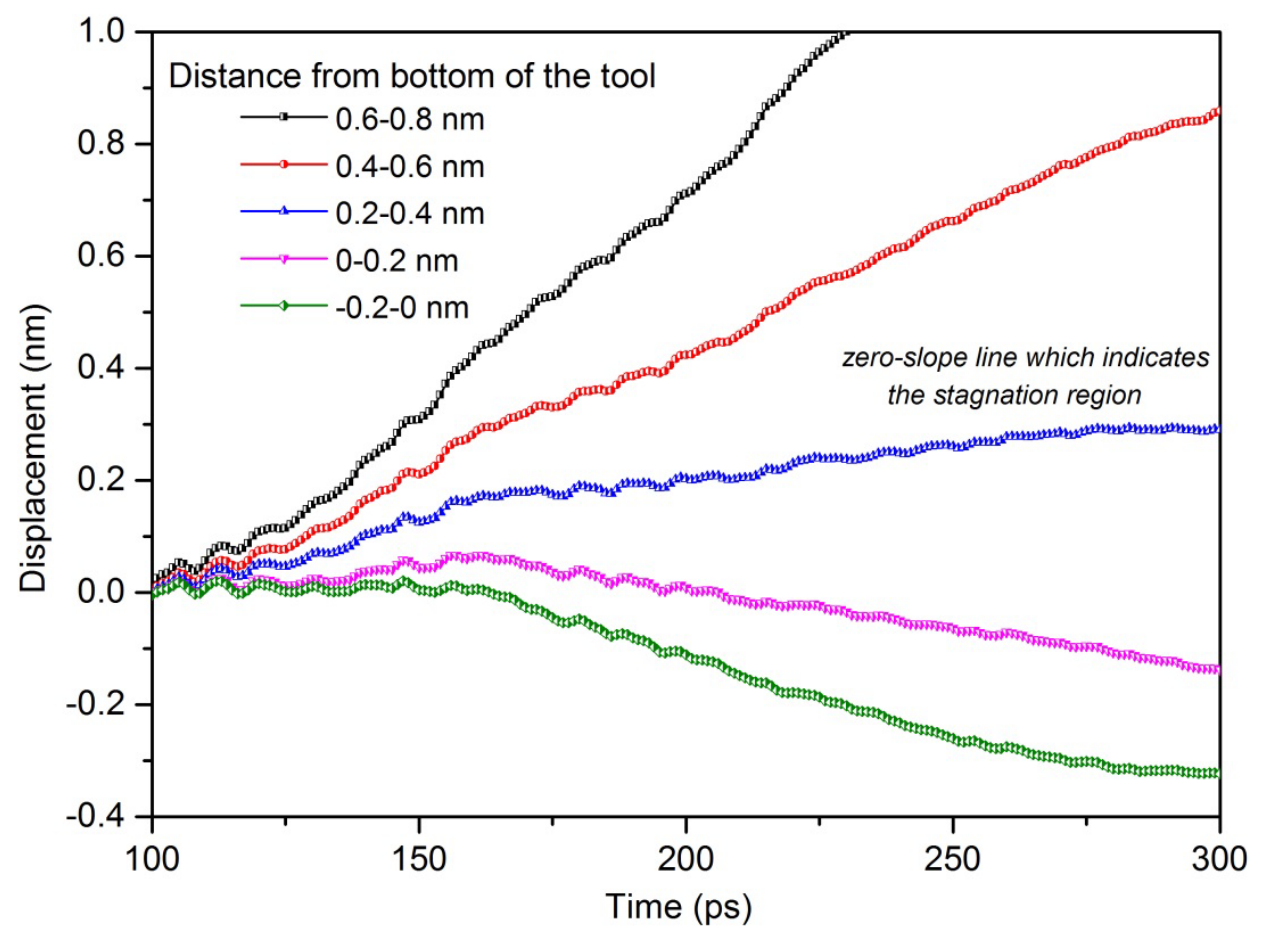

Fig. 6. Displacement of different layers in $y$ direction

In order to locate the stagnation region on the tool tip during the nanometric cutting process, the average displacement of substrate atoms in the $y$ direction in different layers were 
estimated. Fig. 6 shows the quantified displacements of various layers within the substrate while cutting silicon on the (110) crystal plane at 300 K. In Fig. 6, the slope alteration from positive (+) to negative (-) indicates the stagnation region. Thus, the $0.2 \mathrm{~nm}-0.4 \mathrm{~nm}$ region (from bottom of the tool) was identified as the stagnation region for the simulated cutting configuration. Table 1 and Table 2 summarize the position of stagnation region and corresponding angles obtained both by the modified Tersoff and ABOP interatomic potentials, respectively, while cutting silicon on the different crystal planes and at various temperatures. There appears to be a variation in the position of the stagnation region for different crystal orientations and substrate temperatures, particularly when the modified Tersoff potential function was adopted.

In general, when cutting was performed on the (111) plane, the stagnation region (irrespective of the cutting temperature) was observed to locate at an upper position than those observed on the (010) and (110) orientations. This signifies that ploughing due to compression is more on the (111) plane and thus the extent of deformed surface and sub-surface leading to higher spring-back is more pronounced on this orientation. Also, at high temperatures, the stagnation region was observed to shift downwards than what was observed at room temperature. It was interesting to see that the position of stagnation region predicted by the ABOP remains the same for the performed in the temperature range of $750 \mathrm{~K}$ to $1173 \mathrm{~K}$, confirming that $\mathrm{ABOP}$ is not robust to study the flow behaviour of silicon at high temperatures.

Analytical and experimental approaches have shown a clear correlation between stagnation angle and friction angle especially during cutting of soft materials [10]. Therefore, it is instructive at this point to examine the relation between stagnation angle and friction angle in single crystal silicon. The friction angle $(\beta)$ for a rounded edge tool was estimated through equation (2), which uses the effective rake angle in place of nominal rake angle.

$$
\beta=\alpha_{e f}+\tan ^{-1}\left(\frac{F_{c}}{F_{t}}\right)
$$




$$
\alpha_{e f}=\sin ^{-1}\left(1-\frac{d}{r}\right)
$$

where $\alpha_{e f}$ is the effective rake angle, $F_{c}$ is the tangential cutting force, $F_{t}$ is the thrust force and $d$ is the uncut chip thickness. The values of friction angle obtained by both the potential functions have been listed in Table 1 and Table 2.

While cutting silicon on the (111) and (010) at lower machining temperatures viz. $300 \mathrm{~K}$ to 850 $\mathrm{K}$, the magnitude of stagnation and friction angle were observed to be closely related. However, an inconsistency was observed as soon as the machining conditions were changed i.e. on the (110) crystal plane, an average difference of $15^{\circ}-25^{\circ}$ was observed between the two angles. A noteworthy finding is that the discrepancy between stagnation and friction angle increases with the increase of the substrate temperature for the different crystallographic planes.

Table I: Stagnation region, stagnation angle and friction angle in nanometric cutting of single crystal silicon at various temperatures obtained by modified Tersoff potential function

\begin{tabular}{|c|c|c|c|c|}
\hline \multirow[b]{2}{*}{$\begin{array}{c}\text { Substrate } \\
\text { Temperature } \\
\text { (K) }\end{array}$} & \multirow{2}{*}{$\begin{array}{c}\text { Crystal } \\
\text { orientation }\end{array}$} & \multirow[b]{2}{*}{$\begin{array}{c}\text { Position of } \\
\text { Stagnation region from } \\
\text { bottom of the tool } \\
\qquad\left(h_{1,2}\right)(\mathrm{nm})\end{array}$} & \multirow{2}{*}{$\begin{array}{l}\text { Stagnation angles } \\
\left(\theta_{s 1,2}\right) \text { (degree) }\end{array}$} & \multirow[b]{2}{*}{$\begin{array}{l}\text { Friction } \\
\text { angle }(\beta) \\
\text { (degree) }\end{array}$} \\
\hline & & & & \\
\hline \multirow{3}{*}{300} & (010) & $0.5-0.7$ & 31-36.9 & 39.3 \\
\hline & (110) & $0.2-0.4$ & $19.5-27.7$ & 38.6 \\
\hline & (111) & $0.7-0.9$ & $36.9-42$ & 38.3 \\
\hline \multirow{3}{*}{500} & (010) & $0.5-0.7$ & $31-36.9$ & 38.7 \\
\hline & (110) & $0.2-0.4$ & $19.5-27.7$ & 38.9 \\
\hline & (111) & $0.8-1$ & $39.9-44.4$ & 40.6 \\
\hline \multirow[b]{2}{*}{750} & $(010)$ & $0.3-0.5$ & 23.9-31 & 38.2 \\
\hline & (110) & $0.2-0.4$ & $19.5-27.7$ & 36.3 \\
\hline
\end{tabular}




\begin{tabular}{|c|c|c|c|c|}
\hline & $(111)$ & $0.5-0.7$ & $31-36.9$ & 37.1 \\
\hline \multirow{3}{*}{850} & $(010)$ & $0.4-0.6$ & $27.7-34$ & 36.7 \\
\cline { 2 - 5 } & $(110)$ & $0.1-0.3$ & $13.7-23.9$ & 38.2 \\
\cline { 2 - 5 } & $(111)$ & $0.8-1$ & $39.9-44.4$ & 42.8 \\
\hline \multirow{3}{*}{1173} & $(010)$ & $0.2-0.4$ & $19.5-27.7$ & 35.9 \\
\cline { 2 - 5 } & $(110)$ & $0.1-0.3$ & $13.7-23.9$ & 37.7 \\
\cline { 2 - 5 } & $(111)$ & $0.4-0.6$ & $27.7-34$ & 43.2 \\
\hline \multirow{3}{*}{1500} & $(010)$ & $0.2-0.4$ & $19.5-27.7$ & 50.6 \\
\cline { 2 - 5 } & $(110)$ & $0.2-0.4$ & $19.5-27.7$ & 45.7 \\
\cline { 2 - 5 } & $(111)$ & $0.3-0.5$ & $23.9-31$ & 53.1 \\
\hline
\end{tabular}

Table II: Stagnation region, stagnation angle and friction angle in nanometric cutting of single crystal silicon at various temperatures obtained by ABOP potential function

\begin{tabular}{|c|c|c|c|c|}
\hline $\begin{array}{c}\text { Substrate } \\
\text { Temperature } \\
\text { (K) }\end{array}$ & $\begin{array}{l}\text { Crystal } \\
\text { orientation }\end{array}$ & $\begin{array}{c}\text { Position of } \\
\text { Stagnation region from } \\
\text { bottom of the tool } \\
\qquad\left(h_{1,2}\right)(\mathrm{nm})\end{array}$ & $\begin{array}{l}\text { Stagnation angles } \\
\left(\theta_{s 1,2}\right) \text { (degree) }\end{array}$ & $\begin{array}{l}\text { Friction } \\
\text { angle }(\beta) \\
\text { (degree) }\end{array}$ \\
\hline \multirow{3}{*}{300} & (010) & $0.7-0.9$ & $36.9-42$ & 26.5 \\
\hline & (110) & $0.9-1.1$ & $42-46.7$ & 24.8 \\
\hline & (111) & $0.8-1$ & $39.9-44.4$ & 31.6 \\
\hline \multirow{3}{*}{500} & (010) & $0.9-1.1$ & $42-46.7$ & 26 \\
\hline & (110) & $1-1.2$ & $44.4-48.9$ & 25.9 \\
\hline & (111) & $0.8-1$ & $39.9-44.4$ & 31.5 \\
\hline \multirow[b]{2}{*}{750} & (010) & $0.8-1$ & 39.9-44.4 & 26.7 \\
\hline & (110) & $0.8-1$ & $39.9-44.4$ & 23.7 \\
\hline
\end{tabular}




\begin{tabular}{|l|c|c|c|c|}
\hline & $(111)$ & $0.8-1$ & $39.9-44.4$ & 32 \\
\hline \multirow{3}{*}{850} & $(010)$ & $0.8-1$ & $39.9-44.4$ & 26.9 \\
\cline { 2 - 5 } & $(110)$ & $0.8-1$ & $39.9-44.4$ & 23.4 \\
\cline { 2 - 5 } & $(111)$ & $0.8-1$ & $39.9-44.4$ & 31.4 \\
\hline \multirow{5}{*}{1173} & $(010)$ & $0.8-1$ & $39.9-44.4$ & 28.2 \\
\cline { 2 - 5 } & $(110)$ & $0.8-1$ & $39.9-44.4$ & 24 \\
\cline { 2 - 6 } & $(111)$ & $0.8-1$ & $39.9-44.4$ & 31.3 \\
\hline \multirow{3}{*}{1500} & $(010)$ & $0.7-0.9$ & $36.9-42$ & 26.2 \\
\cline { 2 - 6 } & $(110)$ & $0.5-0.7$ & $31-36.9$ & 22.2 \\
\cline { 2 - 6 } & $(111)$ & $0.7-0.9$ & $36.9-42$ & 37.7 \\
\hline
\end{tabular}

Following discussion on machining of silicon, an attempt was made to examine the state of the diamond tool post-machining. This is due to the fact that even though diamond is known as the hardest naturally-occurring substance it can cleave on the (111) plane at room temperature. Furthermore, it has been reported that under confined pressure (3.5 GPa-5 GPa) and at high temperature (>1200 K-1300 K) conditions, diamond shows ductile flow, which is meditated by dislocation glide and twinning [11-12]. During nanometric cutting, cutting edge of the diamond tool experiences high stress and consistent exposure to high temperature conditions that could promote its ductile deformation. The observations witnessed in the above description proves that high machining temperature enhances ductile response of silicon which leads us to anticipate lower tool wear during the high temperature cutting process. It must however be recalled that high heat content is closely associated with the wear of diamond i.e. hot environment can bring about accelerated wear of carbon atoms via diffusion, attrition, adhesion and so on. It was therefore felt necessary to examine this hypothesis during the current 
investigation.

Fig. 7 illustrates the evolution of von Mises stress (using ABOP function) and temperature on the cutting edge of the diamond tool (using modified Tersoff function) during nanometric cutting of single crystal silicon on the (110) crystal plane at different temperatures. The same general pattern was observed for the two other orientations and is therefore not presented here for brevity. Table 3 summarizes the average magnitude of von Mises stress measure, Tresca stress measure and temperature on the cutting edge in all the simulated test cases. Table 3 suggests that the cutting tool experiences least stress and temperature on the (111) orientation compared to others, which makes it an easy cutting plane as has been verified from the experiments [9]. The average values were calculated over the steady state cutting conditions (10 to $25 \mathrm{~nm}$ cutting distance). The general pattern was that the magnitude of von Mises stress and Tresca stress on the cutting edge of the tool decreases with an increase in the machining temperature for the different crystallographic planes. It was seen that the von Mises stress and Tresca stress acting on the cutting edge of the tool at low and high temperatures were in the range of 18 to $26 \mathrm{GPa}$ and 10 to $15 \mathrm{GPa}$, respectively. First-principle calculation suggest that the minimum stress needed to cause structural instability in the diamond structure is around 95 GPa that brings a direct diamond to graphite transformation [13]. Apparently, the assessment of the state of the stress on the cutting edge of the tool is not supportive of a direct diamondgraphite transformation.

A notable observation was that even when the nanometric cutting was simulated at $1500 \mathrm{~K}$, the peak average temperature on the cutting edge remains as low as $\sim 375 \mathrm{~K}$, suggesting that plastic deformation of diamond cutting tool is also unlikely. The question then arises as to what happens to diamond during nanometric cutting of silicon. Some investigations by the authors in this regard were made [4] yet, the post-machining phase of diamond was not found owing primarily due to the unavailability of a robust potential function to predict all the phases of 
carbon. This is another interesting area of further research which the authors are willing to pursue further.
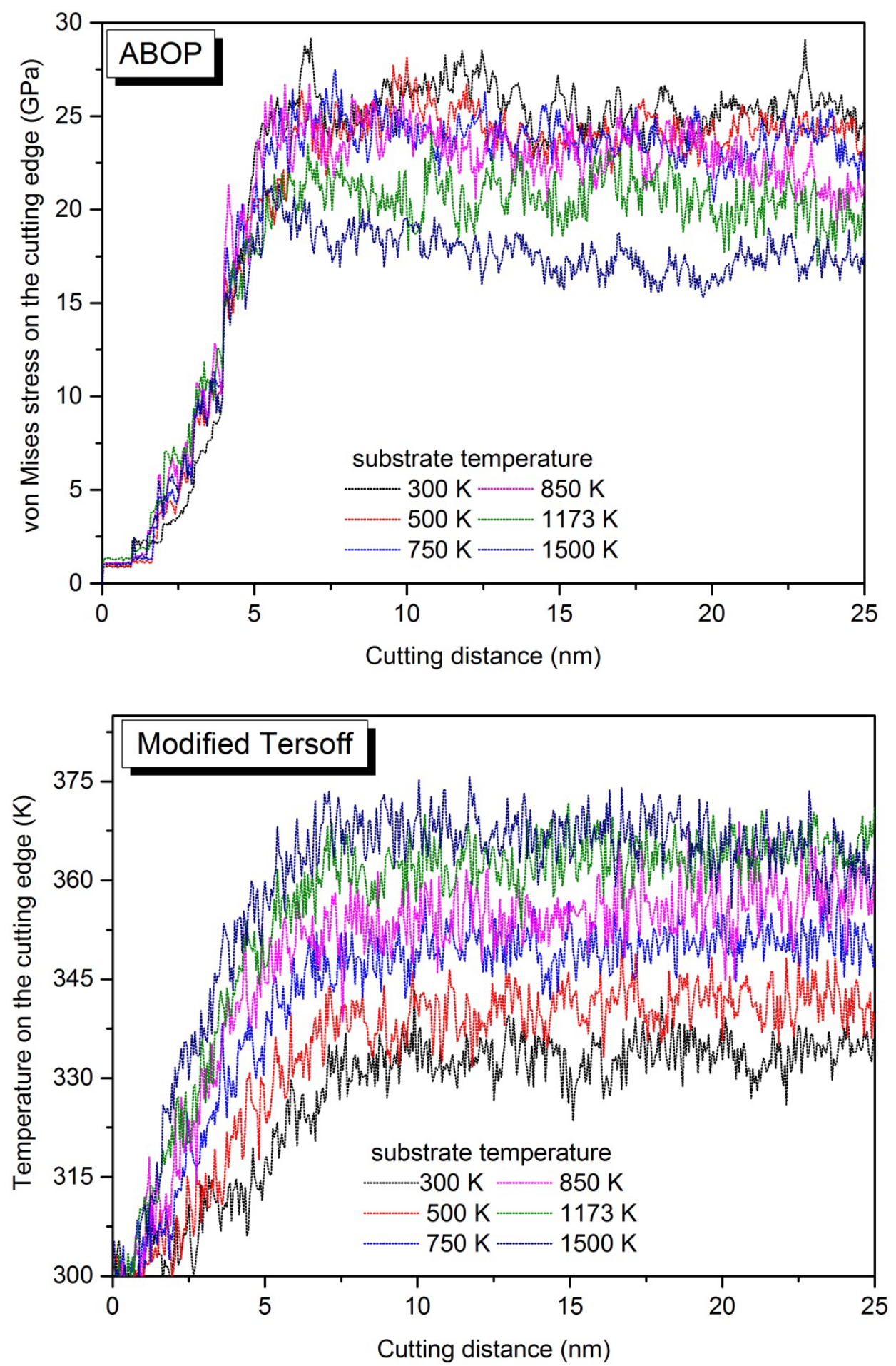

Fig. 7. Evolution of von Mises stress and temperature on the cutting edge of the tool while cutting silicon on the (110) crystal plane 
Table III. Average stresses and temperature on the cutting edge obtained by ABOP and modified Tersoff potential functions, respectively

\begin{tabular}{|c|c|c|c|c|}
\hline $\begin{array}{c}\text { Substrate } \\
\text { Temperature } \\
\text { (K) }\end{array}$ & $\begin{array}{c}\text { Crystal } \\
\text { orientation }\end{array}$ & $\begin{array}{l}\text { von Mises } \\
\text { stress on the } \\
\text { cutting edge } \\
\text { (GPa) }\end{array}$ & $\begin{array}{l}\text { Tresca stress } \\
\text { on the } \\
\text { cutting edge } \\
\text { (GPa) }\end{array}$ & $\begin{array}{l}\text { Temperature } \\
\text { on the } \\
\text { cutting edge } \\
\text { (K) }\end{array}$ \\
\hline \multirow{3}{*}{300} & (010) & 25.2 & 14.1 & 335.6 \\
\hline & (110) & 25.8 & 14.6 & 336.3 \\
\hline & (111) & 24.4 & 13.9 & 329.5 \\
\hline \multirow{3}{*}{500} & $(010)$ & 24.8 & 14 & 344.3 \\
\hline & (110) & 25.4 & 14.1 & 339 \\
\hline & (111) & 23.9 & 13.4 & 337.9 \\
\hline \multirow{3}{*}{750} & (010) & 24.8 & 13.7 & 352.2 \\
\hline & (110) & 23.1 & 13.4 & 347.7 \\
\hline & (111) & 22.7 & 12.1 & 343 \\
\hline \multirow{3}{*}{850} & (010) & 24.1 & 13.7 & 357.3 \\
\hline & (110) & 22.9 & 12.6 & 355.9 \\
\hline & (111) & 22.2 & 12 & 347.1 \\
\hline \multirow{3}{*}{1173} & (010) & 18.8 & 10.8 & 371.2 \\
\hline & (110) & 19 & 10.7 & 367.8 \\
\hline & (111) & 18.4 & 10.3 & 355.8 \\
\hline \multirow{3}{*}{1500} & $(010)$ & 18.6 & 10.5 & 374.9 \\
\hline & (110) & 18.6 & 10.6 & 370.1 \\
\hline & (111) & 18.1 & 10 & 363 \\
\hline
\end{tabular}




\section{Conclusions}

In summary, this work for the first time elucidated flow behaviour of solid silicon in the machining zone during nanometric cutting akin to fluids using a parameter called as atomic displacement vector. MD simulations were performed to reveal that a vortex flow brings higher machinability on the (111) orientation in comparison to the other two orientations. Furthermore, it was observed that the degree of turbulence in the machining zone scales linearly with an increase in the machining temperature. At higher temperatures, elastic recovery is negated by the turbulence, and movement of atoms along the cutting direction was more pronounced. Further analysis showed that the values of the stagnation and friction angle were identical when the cutting was performed on the (111) and (010) crystal planes and at low temperatures. In addition, the stresses and temperature acting on the cutting edge suggest that a direct diamond to graphitic transformation or the plastic deformation of diamond cutting tool is highly unlikely.

\section{Acknowledgment}

The authors gratefully acknowledge the financial support from the EPSRC (EP/K018345/1) grant research for this study. The authors also acknowledge the use of the EPSRC (EP/K000586/1) funded ARCHIE-WeSt High Performance Computer at the University of Strathclyde.

\section{References}

[1] S. Goel, X. Luo, A. Agrawal, R. L. Reuben, Diamond machining of silicon: A review of advances in molecular dynamics simulation, International Journal of Machine Tools \& 
Manufacture 88 (2015) 131-164

[2] S. Plimpton, Fast parallel algorithms for short-range molecular-dynamics. Journal of computational physics, 117 (1) (1995) 1-19

[3] S. Z. Chavoshi, S. Goel, X. Luo, Influence of machining temperature on the anisotropic behaviour of single crystal silicon: A molecular dynamics simulation investigation. Journal of Physics D: Applied Physics. Under review.

[4] S. Goel, X. Luo, R. L. Reuben, Wear mechanism of diamond tools against single crystal silicon in single point diamond turning process. Tribology International 57 (2013) 272-281

[5] P. M. Agrawal, L. M. Raff, R. Komanduri, Monte Carlo simulations of void-nucleated melting of silicon via modification in the Tersoff potential parameters. Physical Review B 72 (2006) 125206

[6] P. Erhart, K. Albe, Analytical potential for atomistic simulations of silicon, carbon, and silicon carbide. Physical Review B, 71(3) (2005) 035211

[7] R. Komanduri, N. Chandrasekarana, L. M. Raff, Molecular dynamics simulation of the nanometric cutting of silicon. Philosophical Magazine Part B 81(12) (2001) 1989-2019

[8] S. Korte, J. S. Barnard, R. J. Stearn, W. J. Clegg, Deformation of silicon-Insights from microcompression testing at 25-500 ${ }^{\circ} \mathrm{C}$. International Journal of Plasticity 27 (2011) 1853-1866

[9] T. Shibata, S. Fujii, E. Makino, M. Ikeda, Ductile-regime turning mechanism of singlecrystal silicon. Precision Engineering 18 (1996) 129-137

[10] M. Malekian, M. G. Mostofa, S. S. Park, M. B. G. Jun, Modeling of minimum uncut chip thickness in micro machining of aluminium. Journal of Materials Processing Technology 212 (2012) 553-559

[11] X. Yu, P. Raterron, J. Zhang, Z. Lin, L. Wang, Y. Zhao, Constitutive law and flow 
mechanism in diamond deformation, Sci. Rep. 2 (876) DOI: 10.1038/srep00876 (2012).

[12] A. Mussi, D. Eyidi, A. Shiryaev, J. Rabier, TEM observations of dislocations in plastically deformed diamond, Phys. Status Solidi A. 210 (2013) 191-194

[13] H. Chacham, L. Kleinman, Instabilities in diamond under high shear stress. Physical Review Letters 85(23) (2000) 4904-4907 